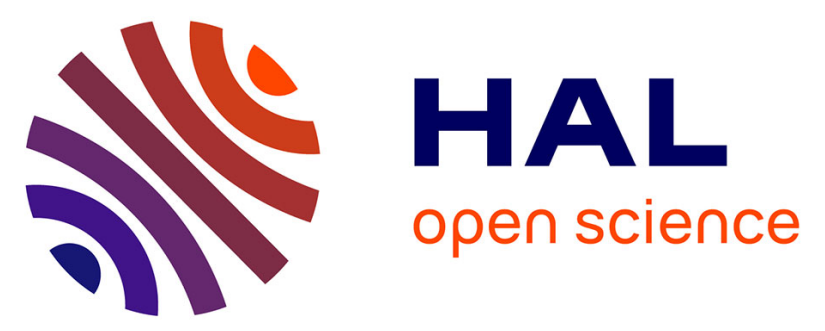

\title{
Dysprosium Single-Molecule Magnets with Bulky Schiff Base Ligands: Modification of the Slow Relaxation of the Magnetization by Substituent Change
}

Jérôme Long, Ivan Basalov, Natalia Forosenko, Konstantin A. Lyssenko, Ekaterina Mamontova, Anton Cherkasov, Marko Damjanović, Liviu Chibotaru, Yannick Guari, Joulia Larionova, et al.

\section{To cite this version:}

Jérôme Long, Ivan Basalov, Natalia Forosenko, Konstantin A. Lyssenko, Ekaterina Mamontova, et al.. Dysprosium Single-Molecule Magnets with Bulky Schiff Base Ligands: Modification of the Slow Relaxation of the Magnetization by Substituent Change. Chemistry - A European Journal, 2019, 25 (2), pp.474-478. 10.1002/chem.201804429 . hal-01980996

\section{HAL Id: hal-01980996 https://hal.science/hal-01980996}

Submitted on 26 Nov 2020

HAL is a multi-disciplinary open access archive for the deposit and dissemination of scientific research documents, whether they are published or not. The documents may come from teaching and research institutions in France or abroad, or from public or private research centers.
L'archive ouverte pluridisciplinaire HAL, est destinée au dépôt et à la diffusion de documents scientifiques de niveau recherche, publiés ou non, émanant des établissements d'enseignement et de recherche français ou étrangers, des laboratoires publics ou privés. 


\title{
Dysprosium Single-Molecule Magnets with Bulky Schiff-base Ligands: Modification of the Slow Relaxation of the Magnetization by Substituent Change
}

\author{
Jérôme Long, ${ }^{*[\mathrm{a}]}$ Ivan V. Basalov ${ }^{[\mathrm{b}]}$ Natalia V. Forosenko, ${ }^{[\mathrm{b}]}$ Konstantin A. Lyssenko, ${ }^{[\mathrm{c}]}$ Ekaterina \\ Mamontova, ${ }^{[a]}$ Anton V. Cherkasov, ${ }^{[b]}$ Marko Damjanović, ${ }^{[d]}$ Liviu F. Chibotaru, ${ }^{[\mathrm{e}]}$ Yannick Guari, ${ }^{[\mathrm{a}]}$ Joulia \\ Larionova, ${ }^{[\mathrm{a}]}$ and Alexander A. Trifonov ${ }^{*[\mathrm{~b}, \mathrm{c}]}$
}

\begin{abstract}
We report the synthesis, magnetic and photoluminescence investigations of two bifunctional dysprosium complexes based on tridentate Schiff base ligands. Magnetic investigations reveal a genuine Single-Molecule Magnet (SMM) behavior with out-of-phase signals up to $60 \mathrm{~K}$, and the tunable emission arising from the Schiff base ligands.
\end{abstract}

Since their discovery fifteen years ago, lanthanide SingleMolecule Magnets (SMM) captivate the chemistry and physics communities due to their possible applications in data storage, spintronics or quantum computing. ${ }^{[1]}$ In such coordination complexes, a slow relaxation of the magnetization, eventually associated with a magnetic bistability, originates from the appearance of an energy barrier, $\Delta$, that opposes two directions of the magnetization. The foundation of such remarkable property lies in the synergistic combination between the intrinsic nature of the lanthanide ions through their angular dependence of the $4 f$ electronic density (oblate vs. prolate) ${ }^{[2]}$ with the crystalfield generated by the surrounding ligands. In this sense, mononuclear lanthanide SMM with tremendous magnetic properties represent a challenge for synthetic chemists in order to design optimized complexes with targeted symmetry and coordination sphere (that includes the electronic density of the donor atoms) in order to maximize the magnetic anisotropy of the $\pm m_{\mathrm{J}}$ states and reduce the Quantum Tunneling of the Magnetization (QTM). This latter produces direct transition between the ground state doublet, however the relaxation of the magnetization could also proceed through other spin-lattice processes (Raman and direct) that decreases the overall magnetic performances through underbarrier pathways.

[a] Dr. J. Long, E. Mamontova, Dr. Y. Guari, Prof. Dr. J. Larionova Institut Charles Gerhardt Montpellier

UMR 5253, Ingénierie Moléculaire et Nano-Objets

ENSCM/CNRS/UM, Place E. Bataillon, 34095 Montpellier Cedex 5 ,

France.

E-mail: jerome.long@umontpellier.fr

[b] Dr. I. V. Basalov, Dr. N. Forosenko, Prof. Dr. A. A. Trifonov Institute of Organometallic Chemistry of Russian Academy of Sciences, 49 Tropinina str., GSP-445, 630950, Nizhny Novgorod, Russia.

E-mail: trif@iomc.ras.ru

[c] Prof. Dr. A. A. Trifonov

Institute of Organoelement Compounds of Russian Academy of Sciences, 28 Vavilova str., 119334, Moscow, Russia.

[e] Dr. M. Damjanović

Physikalisches Institut and Institute of Nanotechnology, Karlsruhe Institute of Technology, Wolfgang-Gaede-Strasse 1, 76131

Karlsruhe, Germany.

[f] Prof. Dr. L. F. Chibotaru

Theory of Nanomaterials Group and INPAC, Katholieke Universiteit Leuven, Celesijnenlaan, 200F, Heverlee, B-3001, Belgium.

Supporting information for this article is given via a link at the end of the document.
Understanding these relaxation mechanisms appears essential to improve the magnetic features. In this sense, since 2016, major breakthroughs in the field have been achieved with examples of mononuclear SMM exhibiting exceptionally large anisotropic barriers of few thousands of $\mathrm{cm}^{-1},[3]$ and a magnetic hysteresis that could almost reach liquid nitrogen's boiling point ${ }^{[3 d, 4]}$ making such molecular systems particularly promising for future technological devices.

Among the possible synthetic strategies to design SMM with large anisotropic barriers, organometallic chemistry appears to be particularly efficient to accurately control the coordination sphere of the lanthanide ion. Such attribute has been efficiently employed to engineer axial crystal-field systems, which can be obtained in linear complexes exhibiting negatively charged ligands along an axis. $\left.{ }^{[3 \mathrm{~d},} 3 \mathrm{e}, 4-5\right]$ This arrangement maximizes the crystal-field splitting for oblate lanthanide ions, while suppressing off-diagonal matrix elements in order to shortcut the QTM. However, examples of such strong axiality in pseudo-linear SMM based on typical coordination chemistry ligands involving nitrogen or oxygen donor atoms are rather scarce $^{[3 a, 3 f, 6]}$ due to the difficulty to realize a linear arrangement and efforts have to be pursued in order to engineer such systems. For instance, Schiff base ligands involving phenoxide moieties have been largely used to obtain lanthanide SMM, ${ }^{[1 b, 7]}$ but the complexes displaying an enhanced axial-crystal field and, as an extent, high energy barriers are rarely met. ${ }^{[8]}$ In this sense, we report in this communication the synthesis and study of two dysprosium Schiff base complexes that display high temperature slow relaxation of their magnetization and luminescence.

Our strategy to obtain Schiff base complexes with a pronounced axial crystal-field relies on using unsymmetrical bulky tridentate ligands, either 2,4-di-tert-butyl-6-((quinolin-8ylimino)methyl)phenolate $\left(L_{1}\right)$ and 2,4-di-tert-butyl-6-(( pyridin-2ylmethyl)imino)methyl)phenolate $\left(L_{2}\right)$ containing the donor set $\mathrm{N}, \mathrm{N}, \mathrm{O}^{-}$in association with the oblate $\mathrm{Dy}^{3+}$ ion. The steric hindrance imposed by tert-butyl groups is favourable to generate mononuclear complexes, while the presence of different pendant groups, such as pyridine or quinoline moieties, will allow tuning the emission wavelength. Thus the reaction of anhydrous $\mathrm{DyCl}_{3}$ with 2 equivalents of in situ prepared potassium phenolates $\mathrm{KL}_{1}$ or $\mathrm{KL}_{2}$ and 1 equivalent of $\mathrm{NaBPh}_{4}$ in THF (Scheme 1) affords the formation of $\left[\mathrm{Dy}\left(\mathrm{L}_{1}\right)_{2}(\mathrm{THF})_{2}\right]\left[\mathrm{B}(\mathrm{Ph})_{4}\right] \quad$ (1) or $\left[\mathrm{Dy}\left(\mathrm{L}_{2}\right)_{2}(\mathrm{py})_{2}\right]\left[\mathrm{B}\left(\mathrm{C}_{6} \mathrm{H}_{5}\right)_{4}\right]$ (2) (see the Supporting Information for details) in 80 and $85 \%$ yield respectively. 


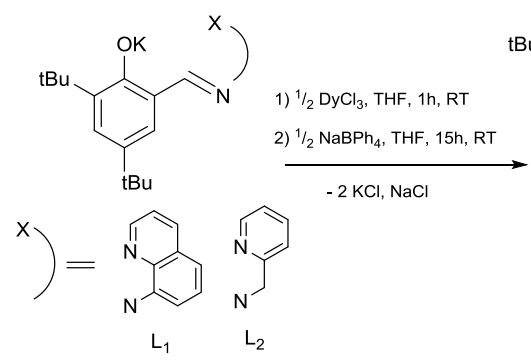

Scheme 1. Synthesis of $\mathbf{1}$ and 2.

X-Ray diffraction on single crystals indicates that both, $\mathbf{1}$ and 2 are cationic mononuclear complexes that crystallize in the chiral $C 222_{1}$ and centrosymmetric $P 2_{1} / n$ space groups, respectively, with a unique crystallographic $\mathrm{Dy}^{3+}$ ion (Figure 1, Table S1). The electroneutrality is ensured by tetraphenylborate anions. The coordination spheres of the Dy ${ }^{3+}$ ions in $\mathbf{1}$ and $\mathbf{2}$ are composed of two deprotonated Schiff base ligands and two coordinated solvate molecules (THF and pyridine for $\mathbf{1}$ and $\mathbf{2}$, respectively) leading to a coordination number of eight. The coordination Dy$\mathrm{N}$ bond lengths in 1 are ranging from $2.503(7)$ to $2.536(6) \AA$. The Dy-O(phenoxide) bond lengths are equal to $2.190(6) \AA$, while the distance involving THF moieties of 2.469(6) $\AA$ are much longer. For 2, the two Dy-O(phenoxide) distances (2.193(2) and 2.196(2) $\AA$ ) are comparable to those in 1. The Dy-N(phenoxide) distances in 2 (2.482(2)-2.548(2) $\AA$ ) are somewhat longer compared to the Dy-N(py) distances (2.594(2), 2.622(2) $\AA$ ). The O-Dy-O angles show the values of 128.5(2) and 121.20(4) ${ }^{\circ}$ for 1 and 2 respectively. The eight-coordinate polyhedrons were quantitatively analysed using the $\mathrm{SHAPE}^{[0]}$ software indicating a biaugmented trigonal prism like geometry of the lanthanide site (Table S2). Thanks to the presence of tetraphenylborate anions, the shortest intermolecular $\mathrm{Dy}^{3+}-\mathrm{Dy}^{3+}$ distances are relatively large (13.959 and $10.658 \AA$ for 1 and 2 respectively) indicating that the complexes are well isolated (Figure S1).

The magnetic properties of complexes 1 and 2 were investigated in both, static and dynamic modes. The room temperature $\chi^{T}$ values of 13.74 and $14.04 \mathrm{~cm}^{3} . \mathrm{K} \cdot \mathrm{mol}^{-1}$ are slightly lower than the value of $14.17 \mathrm{~cm}^{3} \cdot \mathrm{K} \cdot \mathrm{mol}^{-1}$ expected for a single $\mathrm{Dy}^{3+}$ ion, suggesting a significant crystal-field splitting (Figure S2). ${ }^{[10]}$ For temperature lower than $10 \mathrm{~K}$, a dramatic decrease of $\chi T$ is observed confirming the large crystal-field splitting. The field dependence of the magnetization at $1.8 \mathrm{~K}$ shows an inflection point at low field before reaching the values of 5.66 and $5.61 \mathrm{~N} \beta$ under a $70 \mathrm{kOe}$ field (Figure S2). Such sigmoidal shape has been previously observed and imputed to the occurrence of a high anisotropy or magnetic blocking. ${ }^{[3 \mathrm{~b}, 3 \mathrm{e}]}$

The occurrence of a slow relaxation of the magnetization was then investigated using alternate currents (ac) measurements. The frequency dependence of the out-ofphase susceptibilities under a zero dc-field shows a single frequency dependent peak for both compounds (Figure 2, Figure S3). The temperature dependence of the ac susceptibility indicates that out-of-phase signals could be observed up to 60 and $55 \mathrm{~K}$ for 1 and $\mathbf{2}$, respectively (Figure S4).

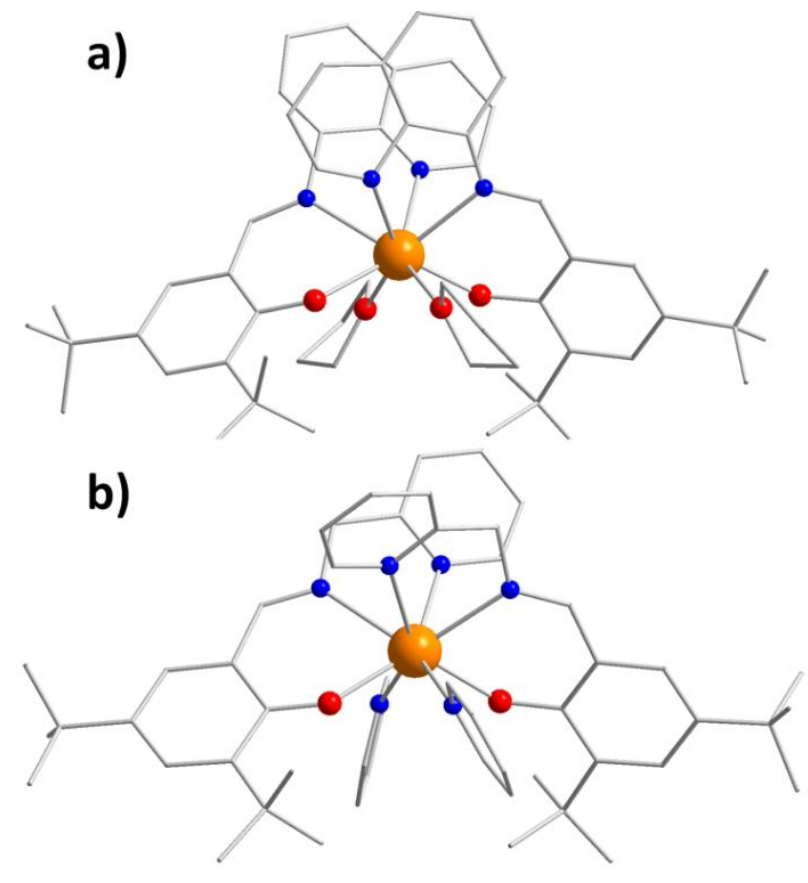

Figure 1. Molecular structure of 1 (a) and 2 (b). Colour code: orange, Dy; blue, $\mathrm{N}$; red, O; grey, C. Hydrogen atoms and $\left[\mathrm{B}(\mathrm{Ph})_{4}\right]^{-}$moieties are omitted for clarity.

For temperature higher than $10 \mathrm{~K}$, fitting of the Cole-Cole plots (Figure S5) with a Debye model confirms the presence of a narrow distribution of relaxation processes with $\alpha$ lower than 0.2 (Table S3-S4).

The relaxation dynamics can be further analyzed by studying the temperature dependence of the relaxation time, $\tau$. The $\ln \tau v s . T^{-1}$ plot (Figure 3) clearly shows a linear region corresponding to a thermally activated relaxation process. However, deviation from linearity is observed for lower temperatures indicating the occurrence of other relaxation processes. Therefore, the whole temperature dependence could be modeled with the following equation $\tau^{-1}=\tau_{0}^{-1} \exp (-\Delta / k T)+C T^{m}+\tau^{-1}$ QTM (Eq. 1)..$^{[11]}$ The first term accounts for an activated process, while the second and third ones stand for two-phonon Raman and QTM, respectively. To avoid over-parameterization, the $m$ coefficient was successfully fixed to integer numbers (1 to 12). The best fitting correlation coefficient was obtained with $m=3$, suggesting the presence of acoustic phonons. ${ }^{[12]}$ The other parameters could be found in Table 1 and indicate that for both compounds, $\Delta$ is found nearly identical. Remarkably, it can be noticed that these values are among the highest reported for mononuclear lanthanide Schiff base complexes.

In order to reduce the QTM, ac measurements were performed under an optimum field of 1000 Oe (Figure S6, Table S5) for both compounds and confirmed the strong decrease of the tunneling rate (Figures S7-9, Table S6-7). The temperature dependence of $\tau$ can be fitted with $\tau^{-1}=\tau_{0}^{-1} \exp (-\Delta / k T)+C T^{m}+$ $A T$ (Eq. 2) (Figure 3, Table S8) in which the third term stands for the direct relaxation process, which may become operative under dc magnetic field. ${ }^{[11]}$ 


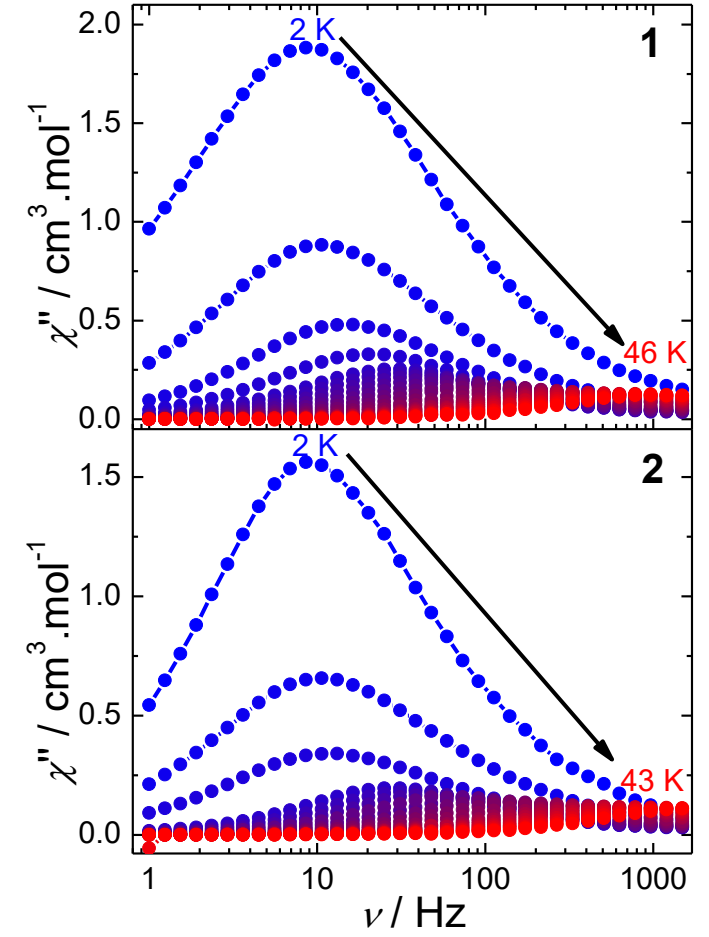

Figure 2. Frequency dependence of the out-of-phase susceptibility, $\chi^{\prime \prime}$, for 1 and $\mathbf{2}$ under a zero dc field.

An important feature for lanthanide SMM concerns also their ability to retain the magnetization. The zero-field cooled (ZFC)/field cooled (FC) curves corroborates the occurrence of a magnetic irreversibility with a maximum of the ZFC curves (corresponding to the blocking temperature $T_{\mathrm{B}}{ }^{[13]}$ ) located at 3.1 $\mathrm{K}$ and $3.0 \mathrm{~K}$ for $\mathbf{1}$ and $\mathbf{2}$, respectively (Figure S10). However, a clear divergence in the ZFC/FC curves, equivalent to an irreversible temperature $\left(T_{\text {irr }}\right)$, could be observed at 8.0 and 5.5 $\mathrm{K}$ for $\mathbf{1}$ and $\mathbf{2}$ respectively which is also concomitant with a crossing between the curves. Such behavior has been previously evidenced in some lanthanide SMM and was interpreted as an out-of-equilibrium state due to the occurrence of competitive relaxation paths. ${ }^{[3 \mathrm{~d}, 3 \mathrm{e}, 4,6,14]}$ Moreover, opening in the hysteresis loops is observed up to 8.0 and $5.0 \mathrm{~K}$ for $\mathbf{1}$ and $\mathbf{2}$, respectively (Figure S11).

In order to get further insights into the mechanisms of slow relaxation, ab initio calculations of the CASSCF level were carried out to quantify the energies of the Kramers doublets, as well as the orientation of the anisotropic axes. The first excited Kramers doublets $\left(m_{\mathrm{J}}= \pm 13 / 2\right)$ are found at 250 and $246 \mathrm{~cm}^{-1}$ (Table S9-S10) for 1 and 2, respectively (Figure 4). These values are in good agreement with those obtained by ac magnetometry suggesting a dominant Orbach relaxation process. This is further confirmed by looking at the $g$ tensor values of the $1^{\text {st }}$ excited state (Table S9-S10) which clearly shows a moderate axiality, explaining the observed anisotropic barriers (Figure 4). In contrast, the $g$ tensor values of the ground Kramers doublet $\left(m_{\mathcal{J}}= \pm 15 / 2\right)$ point out weak transverse anisotropy components but the small difference observed in the $g_{z}$ values (19.85 for 1 vs. 19.78 for 2) explains the lowest tunneling rate (Figure 4) observed in $\mathbf{1}$ and therefore the presence of a magnetic bistability up to higher temperature for this latter. The orientations of the anisotropic axes were also evaluated by ab initio calculations. As expected from the structural analysis, they are slightly tilted with respect to the ODy-O sequence with an easy-axis-Dy-O(phenoxide) angle of $27.6^{\circ}$ for 1 vs. 33.2 and $32.8^{\circ}$ for 2 (Figure S12). The largest crystal-field parameter is observed for $B_{2}^{0}$ in both complexes (Table S11), but it is $11 \%$ greater in magnitude for $\mathbf{1}$, confirming its stronger axiality. While the Dy-O distances are nearly similar for $\mathbf{1}$ and 2, the O-Dy-O angles show a significant difference of about $6 \%$. Remarkably, it could be noticed that the change in the nature of the coordinated solvates (THF vs. pyridine) does not dramatically influence the relaxation dynamic in these complexes. Hence, although THF molecules are stronger coordinated (shorter distances) than pyridine, a greater axiality is observed in 1. Hence, the smaller O-Dy-O angle observed in $\mathbf{2}$ explains its slightly enhanced tunneling rate with respect to 1 .

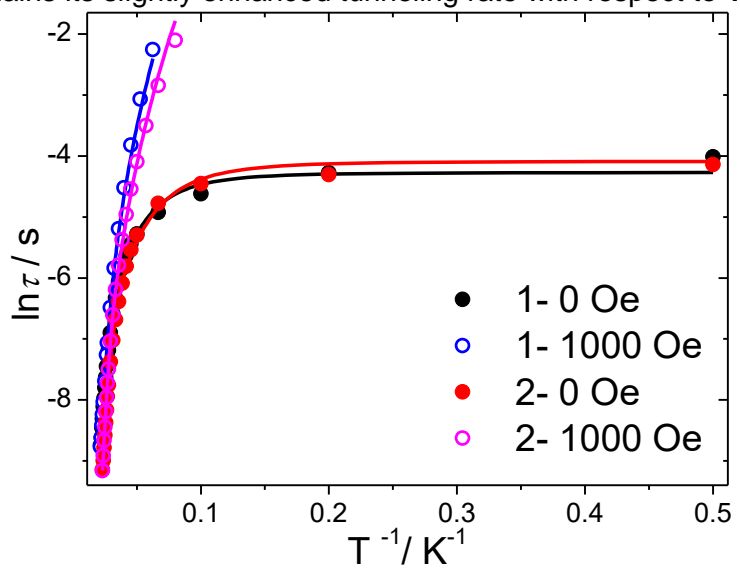

Figure 3. Temperature dependence of the relaxation time using the ac susceptibility data for $\mathbf{1}$ and 2 . The solid lines represent the fit with Eq. 1 ( 0 Oe) or Eq. 2 (1000 Oe).

Table 1. : Fit parameters of the temperature dependence of the relaxation time for 1 and 2.

\begin{tabular}{cccccc}
\hline Compound & $\Delta\left(\mathrm{cm}^{-1}\right)$ & $\tau_{0}(\mu \mathrm{s})$ & $m^{\mathrm{a}]}$ & $C\left(\mathrm{~s}^{-1} \cdot \mathrm{K}^{-\mathrm{m}}\right)$ & $\begin{array}{c}\tau_{\mathrm{QTM}} \\
(\mathrm{ms})\end{array}$ \\
\hline $\mathbf{1}(0 \mathrm{Oe})$ & $263 \pm 8$ & $0.05 \pm 0.01$ & 3 & $0.015 \pm 0.001$ & $14 \pm 1$ \\
$\mathbf{2}(0 \mathrm{Oe})$ & $270 \pm 8$ & $0.015 \pm 0.004$ & 3 & $0.020 \pm 0002$ & $17 \pm 1$ \\
\hline
\end{tabular}

[a] fixed parameter

In addition, Schiff base ligands could also be promising to implement luminescence properties to obtain bifunctional lanthanide that have recently received considerable attention. ${ }^{[15]}$ At room temperature, both, $\mathbf{1}$ and $\mathbf{2}$, exhibit a broad emission band located at 580 and $490 \mathrm{~nm}$, respectively (Figures S13-14). Apart from some changes in the width, these bands resemble those of the $\mathrm{HL}_{1}$ and $\mathrm{HL}_{2}$ ligands (Figure S15) indicating a ligand-based luminescence for $\mathbf{1}$ and $\mathbf{2}$. It was however not possible to observe a dysprosium-centred luminescence, even at low temperature (Figure S16), indicating that these two ligands could not sensitize the Dy ${ }^{3+}$ ion.

To summarize, we have shown that the use of bulky Schiff base ligands involving phenoxide groups could be viewed as an appealing synthetic strategy to design $\mathrm{Dy}^{3+}$-based SMM. The modulation of the substituents directly affects the O-Dy-O angle that in turn alters the QTM. Hence, two genuine SMM with out-of-phase signals up to $60 \mathrm{~K}$ and among the highest 
anisotropic barriers for Schiff base systems have been obtained. Magneto-structural correlations indicate that the control over the axiality of the excited doublets should be achieved to provide relaxation via higher excited states to obtain enhanced magnetic performances. In addition, the compounds exhibit a luminescence originating from the Schiff base ligands which can be tuned depending on substituents making such compounds particularly promising to design multifunctional materials.
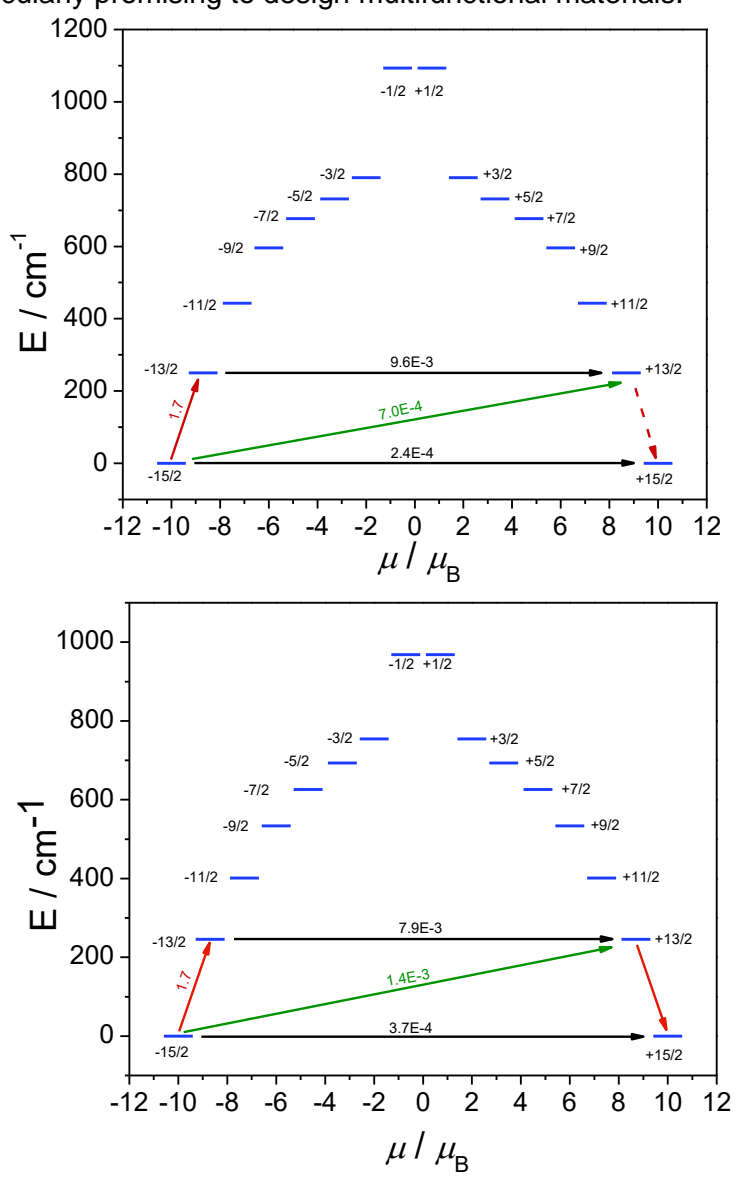

Figure 4. Energy diagrams for the Kramers doublets for 1 (top) and 2 (bottom). The states are placed according to their magnetic moments (bold blue lines). The horizontal black dashed lines show the tunnelling transitions within each doublet state, while the non-horizontal lines show the spin-phonon transition paths. The numbers at non-horizontal lines are averaged transition moments in $\mu_{\mathrm{B}}$ connecting the states. The numbers at horizontal lines are tunnelling gaps. The red dashed arrows delineate the relaxation path.

\section{Acknowledgements}

The authors thank the University of Montpellier, CNRS and PAC of ICGM. The Russian co-authors thank the Russian Science Foundation (grant 17-73-30036). The X-ray study has been carried out using an equipment of The Analytical Center of The G.A. Razuvaev IOMC RAS.

\section{Conflict of Interest}

The authors declare no conflict of interest.

Keywords: Single-Molecule Magnets $\bullet$ Lanthanides $\bullet$ Schiffbases $\cdot$ Luminescence $\cdot$ Dysprosium

[1] a) J. Luzon, R. Sessoli, Dalton Trans. 2012, 41, 13556-13567; b) D. N. Woodruff, R. E. P. Winpenny, R. A. Layfield, Chem. Rev. 2013, 113, 5110-5148; c) F. Troiani, M. Affronte, Chem. Soc. Rev. 2011, 40, 3119 3129; d) L. Bogani, W. Wernsdorfer, Nat. Mater. 2008, 7, 179-186.

[2] J. D. Rinehart, J. R. Long, Chem. Sci. 2011, 2, 2078-2085.

[3] a) J. Liu, Y.-C. Chen, J.-L. Liu, V. Vieru, L. Ungur, J.-H. Jia, L. F. Chibotaru, Y. Lan, W. Wernsdorfer, S. Gao, X.-M. Chen, M.-L. Tong, J. Am. Chem. Soc. 2016, 138, 5441-5450; b) S. K. Gupta, T. Rajeshkumar, G. Rajaraman, R. Murugavel, Chem. Sci. 2016, 7, 51815191; c) L. Ungur, L. F. Chibotaru, Inorg. Chem. 2016, 55, 1004310056; d) F. S. Guo, B. M. Day, Y. C. Chen, M. L. Tong, A. Mansikkamaki, R. A. Layfield, Angew. Chem. Int. Ed. Engl. 2017, 56, 11445-11449; e) Y.-S. Ding, N. F. Chilton, R. E. P. Winpenny, Y.-Z. Zheng, Angew. Chem. Int. Edit. 2016, 55, 16071-16074; f) Y.-S. Meng L. Xu, J. Xiong, Q. Yuan, T. Liu, B.-W. Wang, S. Gao, Angew. Chem. Int. Edit. 2018, 57, 4673-4676.

[4] C. A. P. Goodwin, F. Ortu, D. Reta, N. F. Chilton, D. P. Mills, Nature 2017, 548, 439-442.

[5] T. Pugh, N. F. Chilton, R. A. Layfield, Angew. Chem. Int. Edit. 2016, 55, $11082-11085$

[6] Y.-C. Chen, J.-L. Liu, L. Ungur, J. Liu, Q.-W. Li, L.-F. Wang, Z.-P. Ni, L. F. Chibotaru, X.-M. Chen, M.-L. Tong, J. Am. Chem. Soc. 2016, 138 2829-2837.

[7] a) J. Tang, P. Zhang, in Lanthanide Single Molecule Magnets 10.1007/978-3-662-46999-6_2, Springer Berlin Heidelberg, Berlin, Heidelberg, 2015, pp. 41-90; b) S. T. Liddle, J. van Slageren, Chem. Soc. Rev. 2015, 44, 6655-6669; c) R. A. Layfield, M. Murugesu, Lanthanides and Actinides in Molecular Magnetism, Wiley, 2015.

[8] a) I. Oyarzabal, J. Ruiz, E. Ruiz, D. Aravena, J. M. Seco, E. Colacio, Chem. Commun. 2015, 51, 12353-12356; b) J. P. Costes, S. Titos Padilla, I. Oyarzabal, T. Gupta, C. Duhayon, G. Rajaraman, E. Colacio, Chem. Eur. J. 2015, 21, 15785-15796.

[9] D. Casanova, M. Llunell, P. Alemany, S. Alvarez, Chem. Eur. J. 2005, 11, 1479-1494.

[10] J.-L. Liu, Y.-C. Chen, M.-L. Tong, Chem. Soc. Rev. 2018, 47, 2431 2453.

[11] K. R. Meihaus, S. G. Minasian, W. W. Lukens, S. A. Kozimor, D. K. Shuh, T. Tyliszczak, J. R. Long, J. Am. Chem. Soc. 2014, 136, 60566068.

[12] P. L. Scott, C. D. Jeffries, Phys. Rev. 1962, 127, 32-51.

[13] D. Gatteschi, R. Sessoli, J. Villain, Molecular nanomagnets, Vol. 5 , Oxford University Press on Demand, 2006.

[14] M. Gregson, N. F. Chilton, A.-M. Ariciu, F. Tuna, I. F. Crowe, W. Lewis A. J. Blake, D. Collison, E. J. L. McInnes, R. E. P. Winpenny, S. T. Liddle, Chem. Sci. 2016, 7, 155-165.

[15] a) F. Pointillart, B. le Guennic, O. Cador, O. Maury, L. Ouahab, Acc Chem. Res. 2015, 48, 2834-2842; b) J.-H. Jia, Q.-W. Li, Y.-C. Chen, J.L. Liu, M.-L. Tong, Coord. Chem. Rev. 2017 https://doi.org/10.1016/j.ccr.2017.11.012; c) J. Long, Y. Guari, R. A. S. Ferreira, L. D. Carlos, J. Larionova, Coord. Chem. Rev. 2018, 363, 5770. 
